\title{
NON-LOCAL YIELD LIMIT DEGRADATION
}

\author{
ZDENĚK P. BAŽANT* AND FENG-BAO LIN*
}

Department of Civil Engineering, Northwestern University, Evanston, IL 60208, U.S.A.

\section{SUMMARY}

Presented is a new type of a non-local continuum model which avoids problems of convergence at mesh refinement and spurious mesh sensitivity in a softening continuum characterized by degradation of the yield limit. The key idea, which has recently been proposed in a general context and has already been applied to softening damage due to stiffness degradation, is to apply the non-local concept only to those parameters which cause the degradation while keeping the definition of the strains local. Compared to the previously advanced fully non-local continuum formulation, the new approach has the advantage that the stresses are subjected to the standard differential equations of equilibrium and standard boundary or interface conditions. The new formulation exhibits no zero-energy periodic modes, imbrication of finite elements is unnecessary and finite elements with standard continuity requirements are sufficient. Two-dimensional finite element solutions with up to 3248 degrees of freedom are presented to document convergence and efficacy. The formulation is applied to tunnel excavation in a soil stabilized by cement grouting, with the objective of preventing cave-in (burst) of the tunnel sides due to compression softening.

\section{INTRODUCTION}

As is now well documented, macroscopic description of distributed cracking or other types of damage in various materials requires constitutive models which exhibit strain-softening-a decrease of stress at increasing strain, or in general a loss of positive definiteness of the tangential moduli matrix of the material. This behaviour is observed macroscopically in many materials, including concrete, rocks, stiff soils, grauted soils, two-phase ceramics, various fibre composites, wood and particle board, paper, ice, especially sea ice, coal, filled elastomers, polymer and asphalt concretes, fibre-reinforced concretes, various refractories and metals exhibiting void nucleation and growth in large strains.

The loss of positive definiteness of the elastic moduli matrix was probably first considered by Hadamard ${ }^{28}$ who pointed out that this property is inadmissible from the mathematical viewpoint since the wave speed ceases to be real, the hyperbolic differential equation of motion of the continuum changes its type to an elliptic differential equation, and consequently wave propagation becomes impossible. This argument was later restated and expanded by Hill, Thomas, Mandel, Truesdell and others. ${ }^{5}$ About a decade ago, however, it became clear from experimental results that Hadamard's argument is not quite realistic since real materials in a strain-softening state possess not only a non-positive definite moduli matrix for further loading, but also another incremental moduli matrix for unloading which is always positive definite. Thus, Hadamard's argument about imaginary wave speed applies only to loading waves, while unloading waves can

* Professor of Civil Engineering

† Graduate Research Assistant; presently Assistant Professor of Civil Engineering, Polytechnic University, Brooklyn, N.Y. 11201, USA

002-5918/88/081805-19\$09.50

(C) 1988 by John Wiley \& Sons, Ltd.

Received 7 October 1987

Revised December 1987 
still propagate in a strain-softening material. This fact makes a crucial difference. This is one reason which rendered strain-softening problems mathematically meaningful, even for a local continuum.

Solutions to certain wave propagation problems for a local continuum with strain-softening do exist, and some are unique. ${ }^{2,3}$, 7, 16, 21, 22 Explicit step-by-step finite element solutions converge to these continuum solutions, and appear to do so almost quadratically. It must be noted that, as Sandler ${ }^{54}$ emphasized, problems of this type generally belong to the class of ill-posed initialboundary-value problems. The ill-posedness per se, however, cannot be considered physically objectionable. Ill-posed problems, along with a change of type of the differential equation, are accepted as physically realistic in other branches of physics and engineering, e.g. fluid mechanics. ${ }^{31}$ Thus, it appears that the problem with strain-softening models is physical rather than mathematical.

Noting that a positive stiffness must be considered for unloading in a softening material, Bažant ${ }^{2,3}$ showed in his stability analysis of static strain-softening in a bar that, according to a local continuum model, strain-softening must localize into a region of vanishing volume. The same type of behaviour is exhibited by dynamic wave propagation solutions of strain-softening structures. $6,7,12,21,22$ The consequence of this behaviour is that the structure is predicted to fail with a zero energy dissipation. This is of course physically impossible and represents an unacceptable model.

To prevent such unacceptable behaviour, one must introduce mathematical models called localization limiters, which force the strain-softening region to have a certain minimum finite size and thus ensure the energy dissipation at failure to be finite. The simplest, albeit crudest, localization limiter, which was proposed in $1974^{2}$ and was later adopted in the crack band model, ${ }^{3,6,14}$ consists in limiting the finite element size by a certain minimum value, which represents a material property and is approximately related to the size of inhomogenities in the material. Although this approach appears to yield good results for problems of fracture mechanics type, in which the fracture is localized, it might nevertheless be too crude in that it makes it impossible to resolve the distribution of damage density throughout the failure region, thus precluding precise calculation of the energy dissipation, which is important especially for dynamic problems such as impact.

The most general form of a localization limiter appears to be the concept of non-local continuum. This concept, introduced on the basis of statistical analysis of heterogeneous materials by Kröner, ${ }^{35}$ Krumhanzl, ${ }^{36}$ Kunin, and Levin, ${ }^{5}$ and widely applied by Eringen and Edelen $^{25}$ and others, was proposed for application to strain-softening models in References 4 and 6. At present it is clear that numerous particular forms of non-local formulations are possible. One new form which appears to be simple and effective for the modelling of strain-softening behaviour was recently introduced in References 19,48 and 49 in the context of continuum damage mechanics. The basic idea was that only the softening damage should be treated as nonlocal while the elastic behaviour should be treated as local. Bažant and Pijaudier-Cabot ${ }^{18,19}$ extended this idea further, proposing that, most generally, an efficient model for strain-softening should subject to non-local description only those variables which cause strain-softening and should retain a local definition of strain. Thus the material model may be characterized as a nonlocal continuum with local strain. This concept may be applied to all possible constitutive models with strain softening. Its micromechanics justification has been presented. ${ }^{58}$

Ideally, two kinds of strain softening (or damage) may be distinguished.

1. Degradation of material stiffness, which is caused by microcracking, or by void nucleation and growth. In this case the unloading is ideally a straight line towards the origin (Figure $1(\mathrm{a}))$. 

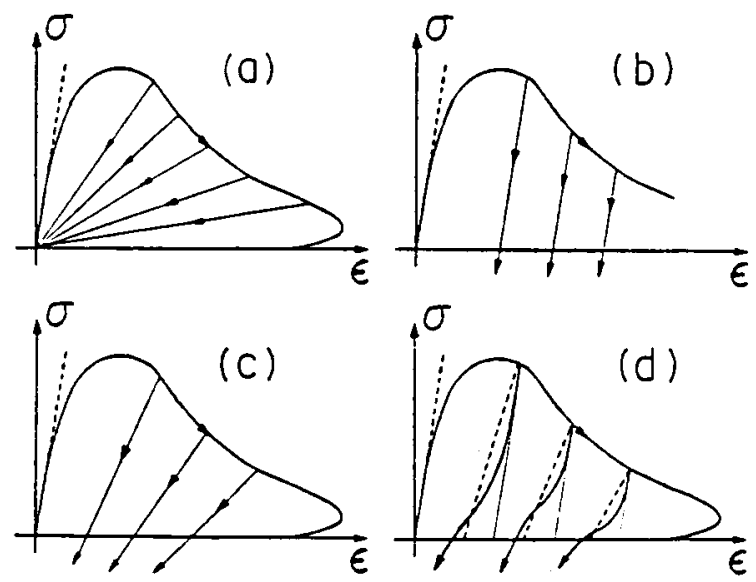

Figure 1. Various types of unloading characteristic of strain-softening materials

2. Degradation of the yield limit, which is associated with the reduction of the areas of cohesive or plastic-frictional connections within the material and does not cause a decrease of material stiffness. In this case the unloading slope is the same as the initial elastic slope (Figure 1(b)).

The real behaviour is better approximated as a combination of the foregoing two basic types (Figure 1(c)). The unloading slope in reality does not remain constant but varies during the progress of unloading (Figure 1(d)). The initial unloading slope is often very close to the initial elastic slope (Figure 1(d)). So, for the initial unloading behaviour, the yield limit degradation (Figure $1(\mathrm{~b})$ ) is a good simplification.

The objective of the present study, which was summarized at a recent conference, ${ }^{13}$ is to formulate for yield limit degradation a non-local continuum model with local strain, verify that it exhibits proper convergence in displacements, stresses and energy, and apply it in finite element analysis of tunnel excavation. As for the degradation of stiffness, a non-local model with local strain has already been developed and demonstrated by finite element examples. ${ }^{15,18,19}$

Before embarking on our analysis, it is proper to mention that localization limiters may be given alternative forms. For example, the averaging integral in the non-local model may be expanded in Taylor series. This leads to higher-order gradients in the definition of strains and in the continuum equations of motion. ${ }^{1,4}$ In particular, higher-order spatial gradients may be applied to the strength limit or yield limit, or to the yield function in general. This type of idea was apparently first proposed in 1952 by L'Hermite ${ }^{42}$ as part of his study of shrinkage cracking. Later the idea of the dependence of strength limit on strain gradient was applied in the problems of bending of concrete beams with earthquake engineering applications. ${ }^{32}$ Recently, the idea of introducing gradients of strength or of the yield condition was developed in the finite element context. $26,43,55,56$

As another alternative, one may avoid the problem of localization by lumping all the softening deformations into a line. This leads to the line crack models with softening non-linear cohesive zone (line segment) ahead of the crack tip (cf. Reference 5), which were pioneered for concrete by Hillerborg with co-workers. ${ }^{29,30}$ This approach is applicable to cracks whose spacing is not less than a certain characteristic length that must be considered to be a material property. ${ }^{5}$ Combined models, in which a line crack or strip is embedded in a larger finite element, were formulated by 
Pietruszczak and Mróz, ${ }^{47}$ Willam et al. ${ }^{57}$ and others. Some authors have criticized the idea of strain-softening on various grounds; ${ }^{51.54}$ however, the criticized aspects have been overcome by the non-local models as well as other mathematical devices.

Much attention has recently been focused on the models of continuum damage mechanics. $^{33,34,37-41,44}$ These models, however, were considered as local, and it has been overlooked that in such a form they are plagued by the same problems of localization (and the associated spurious mesh sensitivity) as the other strain-softening models. ${ }^{8}$ It is nevertheless possible to rectify these problems by incorporating into the continuum damage mechanics formulation the concept of non-local continuum with local strain. ${ }^{19,49}$

As another device to overcome the problems due to change of type of the differential equation, it has been proposed to introduce various forms of strain-rate sensitivity or artificial viscosity. ${ }^{45}$ This seems, though, only a partial remedy, since strain and energy dissipation can localize to an arbitrarily small volume after a sufficient time period. Finally, it should be noted that the localization of strain and damage due to softening, analysed from the stability viewpoint already in 1974 (Bažant ${ }^{2}$ ), is not the same problem as the localization problem analysed by Rudnicki and Rice, ${ }^{53}$ Rice, ${ }^{52}$ Prevost $^{50}$ and others. In those problems, the localization was of primarily geometrically non-linear origin.

\section{NON-LOCAL CONTINUUM WITH LOCAL STRAIN}

The typical property of non-local continuum is that some of the variables in the constitutive equation are defined by spatial averaging. Thus, for example, the spatial average of the magnitude of plastic strain $\varepsilon^{p}$ at location $\mathbf{x}$ may be defined by the equation

$$
\left\langle\varepsilon^{\mathfrak{p}}(\mathbf{x})\right\rangle=\frac{1}{V_{\mathbf{r}}(\mathbf{x})} \int_{V} \alpha(\mathbf{s}-\mathbf{x}) \varepsilon^{\mathrm{p}}(\mathbf{s}) \mathrm{d} V=\int_{V} \alpha^{\prime}(\mathbf{x}, \mathbf{s}) \varepsilon^{\mathrm{p}}(\mathbf{s}) \mathrm{d} V
$$

in which

$$
\begin{gathered}
V_{\mathrm{r}}(\mathbf{x})=\int_{V} \alpha(\mathbf{s}-\mathbf{x}) \mathrm{d} V \\
\alpha^{\prime}(\mathbf{x}, \mathbf{s})=\alpha(\mathbf{s}-\mathbf{x}) / V_{\mathrm{r}}(\mathbf{x})
\end{gathered}
$$

The pointed brackets $\langle>$ denote the averaging operator, $V=$ volume of the body and $\alpha(x)$ $=$ weighting function which defines the averaging; $\mathbf{s}$ is the general co-ordinate vector. $V_{\mathrm{r}}$ has approximately but not exactly the same meaning as the representative volume in the statistical theory of heterogeneous materials (Kröner; ${ }^{35} \mathrm{Krumhansl}^{36}$ ). The averaging may be specified by a uniform weighting function, $\alpha=1$, which is non-zero only within a representative volume such as a circle in two dimensions or a sphere in three dimensions. However, numerical computations show much better convergence if the weighting function is smooth. Thus, a suitable form of the weighting function for numerical computation appears to be, for example, the normal (Gaussian) distribution function (error density function):

$$
\alpha(\mathbf{x})=\mathrm{e}^{-(k|\mathbf{x}| / l)^{2}}
$$

in which, for one, two and three dimensions

$$
\begin{array}{lll}
\text { 1D: } & |\mathbf{x}|^{2}=x^{2}, & k=\sqrt{\pi}=1 \cdot 772 \\
\text { 2D: } & |\mathbf{x}|^{2}=x^{2}+y^{2}, & k=2 \\
\text { 3D: } & |\mathbf{x}|^{2}=x^{2}+y^{2}+z^{2}, & k=(6 \sqrt{\pi})^{1 / 3}=2 \cdot 149
\end{array}
$$


$l$ is the characteristic length, a material property which defines the diameter of the representative volume (a line segment, circle or sphere); $l$ is determined from the condition that the representative volume has the same volume as the normal distribution function extending to infinity $(x, y, z$ $=$ Cartesian co-ordinates).

For a finite body, the normal distribution function extends beyond the boundary of the body. In that case the region outside the body is deleted from the integration domain $V$, both for the calculation of the average (equation (1)) and the calculation of the representative volume $V_{\mathrm{r}}$ (equation (3)). This fact causes $V_{\mathrm{r}}$ to depend on location $\mathbf{x}$.

For numerical finite element computations, the integrals in equations (1)-(3) are approximated by finite sums over all the integration points of all the elements. Only the finite elements whose integration points are closer to point $\mathbf{x}$ than distance $2 l$ need to be included in the sum since for a greater distance the value of $\alpha$ is negligible.

In the initial application of the non-local concept to strain-softening, ${ }^{4}$ it was assumed that the strain was non-local. In such a case the variational derivation of the differential equations of motion or equilibrium and of the boundary conditions from the virtual work principle ${ }^{4}$ yields field equations and boundary conditions of a non-standard form, with extra terms in the form of integrals or higher-order derivatives. This aspect causes considerable complications in numerical implementation. Recently ${ }^{18,19,48}$ it was discovered that application of the averaging operator to strains is not necessary, and that a sufficient localization limiter may be obtained if the non-local averaging is applied only to those variables which control strain-softening. In that case the statement of the virtual work principle is the same as for the classical continuum, from which it has been mathematically proved that the differential equations of motion or equilibrium with their boundary conditions as well as interface conditions remain the same as in the classical theory (see Bažant and Pijaudier-Cabot ${ }^{18,19}$ ). Consequently, the continuity requirements for the finite elements are the same as for a local finite element model (and no element imbrication, introduced in the initial non-local model for strain-softening, is needed). It has also been generally proved that the damage dissipation, which is controlled by the variables that govern softening, cannot localize to a region of vanishing volume. ${ }^{19}$

\section{REVIEW OF CLASSICAL INCREMENTAL PLASTICITY}

First we must define the local plasticity model which we want to generalized to non-local form. The yield surfaces are written as $F(\sigma, \kappa)=0$ and the yield function is assumed to have the form $F(\sigma, \kappa)=f(\sigma)-\kappa$, in which $\sigma=$ stress tensor; function $f(\sigma)$ may be regarded as the effective stress $\sigma^{*}$, and $\kappa$ is a hardening-softening parameter which may be interpreted as the yield limit; $\kappa$ may in general not only increase (hardening) but also decrease (softening). We assume for the sake of simplicity that there is only one hardening-softening parameter. As usual, we require the increments $\Delta \varepsilon^{p}$ of the plastic strain tensor $\varepsilon^{p}$ to follow the normality rule

$$
\Delta \varepsilon^{\mathrm{p}}=F_{, \mathrm{\sigma}} \Delta \lambda=f_{, \mathrm{\sigma}} \Delta \lambda
$$

where a comma preceding a subscript denotes a derivative, i.e. $F_{, \sigma}=\partial F / \partial \sigma$, and $\Delta \lambda$ represents a scalar proportionality coefficient. The loading-unloading criterion is defined by the conditions

$$
F \Delta \lambda=0, \quad \Delta \lambda \geqslant 0, \quad F \leqslant 0
$$

which must all hold simultaneously.

We may now assume that $\kappa$ depends on $\varepsilon^{\mathfrak{p}}$ but not directly on $\sigma$, and introduce Prager's continuity condition

$$
F_{, \boldsymbol{\sigma}}: \Delta \boldsymbol{\sigma}+F_{, \kappa} \kappa_{, \mathbf{x}^{\mathrm{p}}}: F_{, \mathbf{\sigma}} \Delta \lambda=0
$$


where the colon (:) denotes a tensor product contracted on two inner indices. We may also denote

$$
H=-F_{, \kappa} \kappa_{, \varepsilon p} \varepsilon_{, \varepsilon^{p}}^{\mathrm{p}}: F_{, \sigma}
$$

where $H$ may be called the plastic tangential modulus and $\kappa$ is assumed to depend on $\varepsilon^{\mathrm{p}}$. Thus the continuity condition becomes $F_{, \sigma}: \Delta \sigma-H \Delta \lambda=0$, i.e. $\Delta \lambda=\left(F_{, \sigma}: \Delta \sigma\right) / H$. Substituting this and equation (8) into the incremental stress-strain relation

$$
\Delta \sigma=\mathbf{D}:\left(\Delta \varepsilon-\Delta \varepsilon^{p}\right)
$$

where $\mathbf{D}$ is the tensor of elastic moduli, we may solve for $\Delta \lambda$ and obtain the well-known relation ${ }^{46}$

$$
\Delta \lambda=\frac{F_{, \sigma}: \mathrm{D}: \Delta \varepsilon}{F_{, \sigma}: \mathrm{D}: F_{, \sigma}+H}
$$

Usually the effective plastic strain either is considered as the path length of plastic strain, i.e. $\Delta \varepsilon^{\mathfrak{p}}=\left(\Delta \varepsilon^{\mathfrak{p}}: \Delta \varepsilon^{\mathrm{p}}\right)^{1 / 2}$, or defined by work equivalence $\sigma: \Delta \varepsilon^{\mathrm{p}}=\sigma^{*} \Delta \varepsilon^{\mathrm{p}}$. We choose the latter definition, and then

$$
\Delta \varepsilon^{\mathrm{p}}=\frac{1}{\sigma^{*}} \sigma: F_{, \sigma} \Delta \lambda
$$

The yield function can be written in the form $F(\sigma, \kappa)=f(\sigma)-\kappa$; function $f(\sigma)$ may normally be assumed to be a homogeneous function of degree $n$, for which Euler's theorem states that $\sigma: f_{, \sigma}=n f$. Furthermore, the yield function may be written in such a form that $n=1$ and $f(\sigma)=\sigma^{*}$. Also, the hardening-softening parameter $\kappa$ may be assumed to be a function of the effective plastic strain $\varepsilon^{\mathrm{p}}$ rather than a general scalar function of $\varepsilon^{\mathrm{p}}$, i.e. $k=k\left(\varepsilon^{\mathrm{p}}\right)$. Then the aforementioned expression for $H$ may be further simplified as follows:

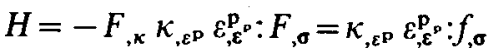

$$
\begin{aligned}
& =\kappa_{, \varepsilon^{\mathrm{p}}} \frac{1}{\sigma^{*}}\left(\sigma: f_{, \sigma}\right)=\kappa_{, \varepsilon^{\mathrm{p}}} \frac{n f}{\sigma^{*}}=\kappa_{, \varepsilon^{\mathrm{p}}}
\end{aligned}
$$

\section{NON-LOCAL GENERALIZATION}

According to the concept of non-local continuum with local strain, ${ }^{19,56}$ equation (10) must be generalized as

$$
\Delta \boldsymbol{\sigma}=\mathbf{D}:\left(\Delta \varepsilon-\Delta \bar{\varepsilon}^{\mathrm{p}}\right)
$$

in which $\Delta \varepsilon$ remains to be local while $\Delta \bar{\varepsilon}^{\mathrm{p}}$ is the non-local plastic strain increment. Note that we distinguish the superimposed bar, which is a label for non-local quantities, from the pointed brackets \langle\rangle , which represent an operator.

We now present the algorithm of the initial stiffness method for an implicit finite element solution with step-by-step loading. The computations in each loading step proceed as follows.

1. The calculation begins by carrying out an elastic finite element analysis of the structure using the applied load increments $\Delta \mathbf{f}$ (or prescribed displacement increments) and the nodal forces $\Delta \mathbf{f}^{\prime \prime}$. For the first iteration, $\Delta \mathbf{f}^{\prime \prime}$ are taken as zero. For the subsequent iterations, forces $\Delta \mathbf{f}^{\prime \prime}$ are determined from the previous iteration of this loading step; they represent the nodal forces equivalent (according to the principle of virtual work) to the unbalanced stresses determined in step 6 of this algorithm (defined below). This finite element analysis yields $\Delta \mathbf{u}$ and $\Delta \varepsilon$, where $\Delta \mathbf{u}$ is 
the column matrix of all nodal displacement increments and $\Delta \varepsilon$ is the corresponding column matrix of strain increments (for all integration points of all finite elements). The elastic stress increments are obtained as

$$
\Delta \boldsymbol{\sigma}^{\mathrm{e}}=\mathbf{D}: \Delta \varepsilon
$$

and the final stress values are $\sigma_{\mathrm{F}}=\sigma_{1}+\Delta \sigma^{\mathrm{e}}$, where subscripts I and $\mathrm{F}$ refer to the initial and final values for the loading step.

2. Next we calculate for all the integration points of all the elements the values $F_{F}$ $=F\left(\sigma_{\mathrm{l}}+\Delta \sigma^{\mathrm{e}}, \kappa_{\mathrm{F}}\right)$, where $\kappa_{\mathrm{F}}$ is the final value of $\kappa$ for the loading step and is taken for the first iteration as $\kappa_{\mathrm{F}}=\kappa_{\mathrm{l}}$, and for subsequent iteration as the final $\kappa$-value from the preceding iteration. If $F_{F}<0$, the material at the integration point is still in the elastic range or unloaded.

3. Now $F_{F}>0$. This means that the end value of stress is outside the loading surface and must therefore be adjusted to the loading surface. First we need to determine the ratio $r(0<r<1)$ such that $F\left(\sigma_{1}+r \Delta \sigma^{\mathrm{e}}, \kappa_{\mathrm{F}}\right)=0$. Approximately, by linear interpolation, $F_{1}+r\left(F_{\mathrm{F}}-F_{1}\right)=0$, from which we calculate $r=F_{\mathrm{I}} /\left(F_{\mathrm{F}}-F_{\mathrm{I}}\right)$ for all integration points of all elements.

4. To take into account the plastic strain increments, we calculate (for all the integration points of all elements) the value of $H$ according to equation (13). For $F_{, \sigma}, \kappa_{, \varepsilon^{p}}$ and $\varepsilon_{, \varepsilon^{p}}^{p}$ we use the values corresponding to $\sigma_{1}$ and $\varepsilon_{p}^{p}$ for the first iteration, and to $\sigma_{1}+\Delta \sigma$ and $\varepsilon_{1}^{p}+\Delta \varepsilon^{p}$ for the subsequent iterations, with $\Delta \sigma$ and $\Delta \varepsilon^{p}$ taken from the preceding iteration. The strain increments corresponding to the previously determined stress increments $(1-r) \Delta \sigma^{\mathrm{c}}$ are $(1-r) \Delta \varepsilon$. Thus, according to equation (11), we calculate

$$
\Delta \lambda=\frac{F_{, \mathbf{\sigma}}: \mathbf{D}:(1-r) \Delta \varepsilon}{F_{, \boldsymbol{\sigma}}: \mathbf{D}: F_{, \sigma}+H}
$$

for all the integration points of all elements. These are still the local $\Delta \lambda$ values. $F_{, \sigma}$ are here evaluated for $\sigma_{1}$ for the first iteration and for $\sigma_{1}+\Delta \sigma$ for the subsequent iterations using always $\Delta \sigma$ from the preceding iteration. The corresponding local plastic strain increments are then calculated as $\Delta \varepsilon^{\mathfrak{p}}=F,{ }_{, \sigma} \Delta \lambda$ (equation (8)), for all the integration points of all elements.

5. Now comes the non-local operation which yields for each integration point of each element the average non-local plastic strain increment:

$$
\left\langle\Delta \varepsilon^{\mathrm{p}}(\mathbf{x})\right\rangle=\int_{V} \alpha^{\prime}(\mathbf{x}, \mathbf{s}) \Delta \varepsilon^{\mathrm{p}}(\mathbf{s}) \mathrm{d} V \approx \sum_{j} \alpha_{(i, j)}^{\prime} \Delta \varepsilon_{(j)}^{\mathrm{p}}
$$

The last expression is a numerical approximation of the integral. The subscripts in parentheses refer to the integration points of all finite elements in the system, and $\alpha_{(i, j)}^{\prime}$ denotes the values of the function $\alpha^{\prime}(\mathbf{x}, \mathbf{s})$ for the integration point $\mathbf{x}_{(j)}$ (of this or another element) relative to point $\mathbf{x}_{(i)}$, i.e. $\alpha_{(i, j)}^{\prime}=\alpha\left(\mathbf{x}_{(j)}-\mathbf{x}_{(i)}\right) / V_{\mathrm{r}(i)}$. Note that, for sufficiently large $\left|\mathbf{x}_{(j)}-\mathbf{x}_{(i)}\right|$, i.e. for sufficiently remote integration points, the value of $\alpha_{(i, j)}^{\prime}$ can be neglected because the normal probability function decays with distance very rapidly. For numerical implementation, it is convenient to evaluate all the coefficients $\alpha_{(i, j)}^{\prime}$ in advance of the finite element solution and store them as a two-dimensional array in the computer's memory.

6. The definition of the non-local equivalent plastic strain increment $\Delta \bar{\varepsilon}^{\mathrm{p}}$ may be considered similar to equation (12), i.e. $\sigma^{*} \Delta \bar{\varepsilon}^{\mathrm{p}}=\sigma:\left\langle\Delta \varepsilon^{\mathrm{p}}\right\rangle$. Accordingly, we evaluate for each integration point of each element

$$
\Delta \bar{\varepsilon}^{\mathfrak{p}}=\frac{1}{\sigma^{*}} \boldsymbol{\sigma}:\left\langle\Delta \varepsilon^{\mathfrak{p}}\right\rangle
$$


and define non-local $\Delta \overline{\hat{\lambda}}$ by the relation $\sigma: F_{\sigma} \Delta \bar{\lambda}=\sigma:\left\langle\Delta \varepsilon^{p}\right\rangle$. Then we may evaluate for each integration point of each element

$$
\Delta \bar{\lambda}=\frac{\sigma:\left\langle\Delta \varepsilon^{p}\right\rangle}{\sigma: F_{, \sigma}}=\frac{\sigma^{*} \Delta \bar{\varepsilon}^{p}}{\sigma: F_{, \sigma}}
$$

These non-local values differ from the local $\Delta \lambda$ given by equation (16) and are not equal to $\langle\Delta \lambda\rangle$. Also, note that equation (19) does not imply $\left\langle\Delta \varepsilon^{p}\right\rangle$ to be equal to $F_{. \sigma} \Delta \lambda$. Therefore, the nonlocal plastic strain increments $\Delta \bar{\varepsilon}^{\mathrm{p}}$ which are normal to the loading surface may now be obtained for all the integration points of all elements as

$$
\Delta \bar{\varepsilon}^{\mathrm{p}}=F_{.,} \Delta \bar{\lambda}
$$

The final stresses $\sigma_{\mathrm{F}}^{\prime}$ for the loading step are first estimated as

$$
\sigma_{\mathrm{F}}^{\prime}=\sigma_{1}+r \Delta \boldsymbol{\sigma}^{\mathrm{e}}+\mathrm{D}:\left[(1-r) \Delta \varepsilon-\Delta \bar{\varepsilon}^{\mathrm{p}}\right]
$$

in which $(1-r) \Delta \varepsilon$ corresponds to the stresses outside the yield surface. Next we obtain the final value $\kappa_{\mathrm{F}}=\kappa\left(\bar{\varepsilon}_{\mathrm{F}}^{\mathrm{p}}\right)$, where $\bar{\varepsilon}_{\mathrm{F}}^{\mathrm{p}}=\bar{\varepsilon}_{\mathrm{p}}^{\mathrm{p}}+\Delta \bar{\varepsilon}^{\mathrm{p}}=$ final value of the equivalent plastic strain. The $\sigma_{\mathrm{F}}^{\prime}$ from equation (21) does not lie on the yield surface. We apply the radial return method (Figure 2) to adjust it to the yield surface and get the correct final stresses $\sigma_{F}$. The differences between $\sigma_{1}+\Delta \sigma^{\mathrm{e}}$ and $\sigma_{\mathrm{F}}$ represent the unbalanced parts of the elastic stress increments, and must therefore be balanced by applying additional residual forces. This is done later in step 1 of the next iteration. The objective of the iterations of the loading steps is to make the residual forces approximately vanish.

7. This is approximately achieved by imposing the condition $\sum$ (residual force) ${ }^{2}<\varepsilon_{1}$, where the sum is over every nodal point and $\varepsilon_{1}$ is a specified small number representing the tolerance. If the tolerance is not satisfied, then we return to step 1 and start the next iteration of the same loading step. If it is satisfied we also return to step 1 but start the first iteration of the next loading step.

The loading-unloading criterion which is implied by steps 2,3 and 6 of the foregoing non-local algorithm may be concisely stated as

$$
F \Delta \bar{\lambda}=0, \quad \Delta \bar{\lambda} \geqslant 0, \quad F \leqslant 0
$$

As an alternative to the foregoing algorithm, we may apply the averaging operation directly to $\Delta \lambda$ instead of $\Delta \varepsilon^{\mathrm{p}}$. This is computationally more efficient since at each point there are many components of $\Delta \varepsilon^{p}$ but only one $\Delta \lambda$-value. In this alternative, instead of equation (19) we calculate for each integration point of each element the non-local value as

$$
\Delta \bar{\lambda}=\langle\Delta \lambda\rangle=\left\langle\frac{\boldsymbol{\sigma}: \Delta \varepsilon^{\mathbf{p}}}{\boldsymbol{\sigma}: F_{, \boldsymbol{\sigma}}}\right\rangle=\sum_{j} \alpha_{(i, j)}^{\prime}\left(\frac{\boldsymbol{\sigma}: \Delta \varepsilon^{\mathbf{p}}}{\boldsymbol{\sigma}: F_{, \sigma}}\right)_{(j)}
$$

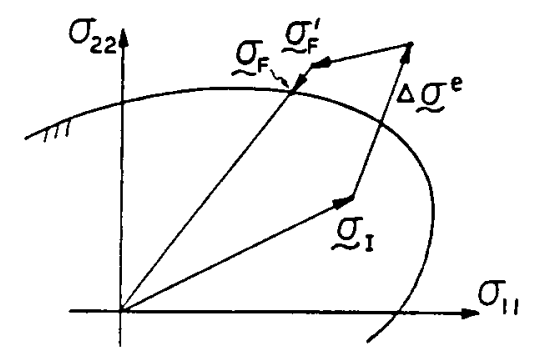

Figure 2. Radial return method for plastic analysis 
The non-local plastic strain increments then are $\Delta \bar{\varepsilon}^{p}=F_{, \sigma} \Delta \bar{\lambda}$ (equation (20)) and the non-local effective plastic strain increment is $\Delta \bar{\varepsilon}^{\mathrm{p}}=\left(\sigma: \Delta \bar{\varepsilon}^{\mathrm{p}}\right) / \sigma^{*}$, from which we get the value of $h\left(\bar{\varepsilon}_{\mathrm{F}}^{\mathrm{p}}\right)$. The remaining equations and the computational algorithm are the same as before. This alternative has been found to yield about the same numerical results as the originally described formulation with the averaging of $\Delta \varepsilon^{\mathfrak{p}}$, and appeared to converge equally well.

\section{EXAMPLE I. DAMAGE BANDS IN TENSIONED RECTANGULAR PANEL}

Figure 3 shows the example of a rectangular panel subjected to uniform prescribed displacement on top boundary and fixed at the bottom boundary, with the lateral boundaries free. The problem is solved with three rectangular meshes using four-node quadrilateral finite elements. In order to initiate a softening band symmetrically, it is assumed that one element at the middle of each side has the initial yield limit smaller by 5 per cent than the remaining finite elements. The side of the finite element in the crudest mesh is assumed to be equal to the characteristic length $l$ which figures in equation (4). The material is assumed to obey Tresca yield condition (Figure 4(b)), and the hardening-softening rule is specified in such a manner that after the attainment of the initial yield limit $\kappa_{0}$ the value of $\kappa$ decreases linearly as a function of $\varepsilon^{\mathrm{p}}$, with a slope $H$, until a zero value of $\kappa$ is reached (Figure $4(\mathrm{~b})$ ). After that $\kappa$ remains zero. Unloading and reloading are elastic.

Figures 4(a) and (b) show the curves of the reaction force $P$ on top as a function of displacement $u$ enforced at the top boundary. The results in Figure 4(a) are local, and those in Figure 4(b) are non-local, based on the present model. It is clear that the local response shows strong spurious mesh sensitivity in the softening range. Were the mesh refined further, the softening response would result in a snapback instability, i.e. the softening response after the peak point would revert to a positive slope. For the present non-local formulation, the results for the three meshes are approximately the same, and especially those for meshes 2 and 3 are nearly identical. This documents that the presently proposed non-local formulation eliminates the phenomenon of spurious mesh sensitivity which has plagued previous uses of strain-softening in finite element computation.

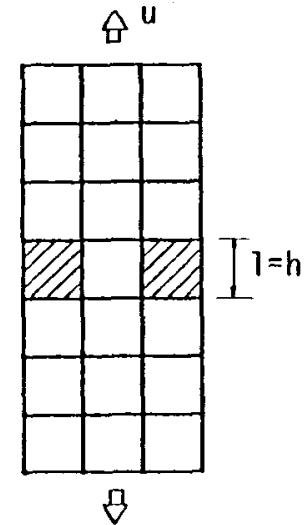

Mesh 1

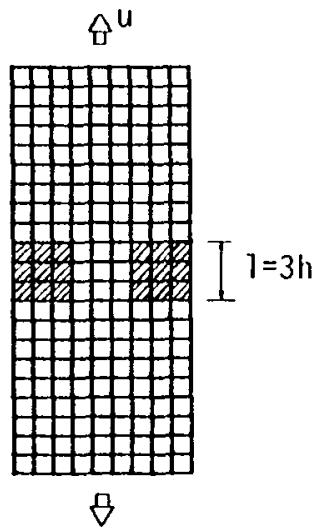

Mesh 2

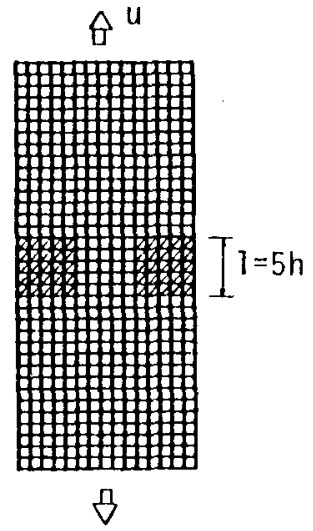

Mesh 3

Figure 3. Rectangular panel with various mesh subdivisions 

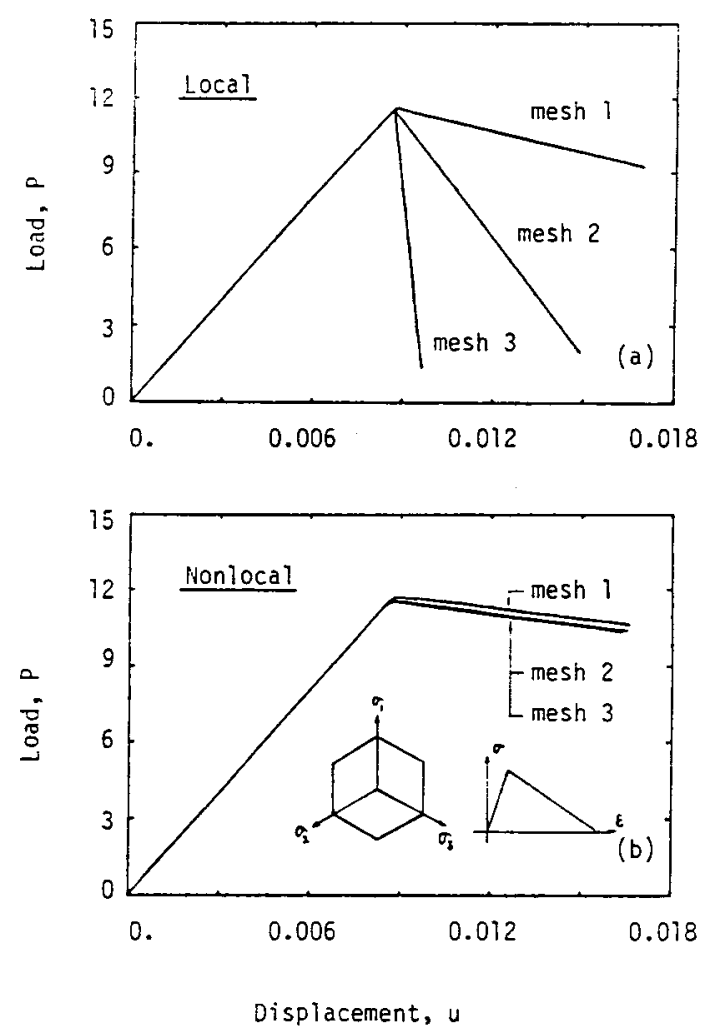

Figure 4. Load deflection diagrams calculated with various meshes according to the local and non-local plasticity model

The strain profiles across the cracking zone are shown in Figure 5. For the local case, the zones are seen to localize into sharper spikes when the mesh is refined. The most important aspect of strain-softening is the energy dissipation. As shown previously for the local solution, the energy dissipated due to strain-softening exhibits strong spurious mesh sensitivity due to localization of the softening front, and generally tends to zero as the mesh is refined to zero. This is documented for the local computation in Figure 6(a). With the present non-local model, by contrast, the energy dissipation appears to converge; see Figure $6(\mathrm{~b})(N=$ number of elements in the mesh, and $W=$ energy dissipated up to the instant at which the displacement on top is 0.025 ).

The softening zones at various stages of loading (for $u=0.00854,0.00868$ and 0.00889 ) are plotted in Figure 7. Note that for the refined meshes the width of the softening zone is not a constant but varies. As a consequence of this fact, the energy dissipation rate per unit length of the strain-softening band is also not a constant but varies during the progress of loading. This has an interesting implication for non-linear line crack fracture models with a cohesive zone, such as Hillerborg's model for concrete: the fracture energy which would match the present results could not be considered as constant but would have to be variable. Moreover, due to the variation of the width of the softening band, the non-local solution cannot be equivalent to a line crack solution with a unique stress-displacement diagram. Rather, a different stress-displacement diagram would have to be considered for various stages of computation in order to match the present results. 

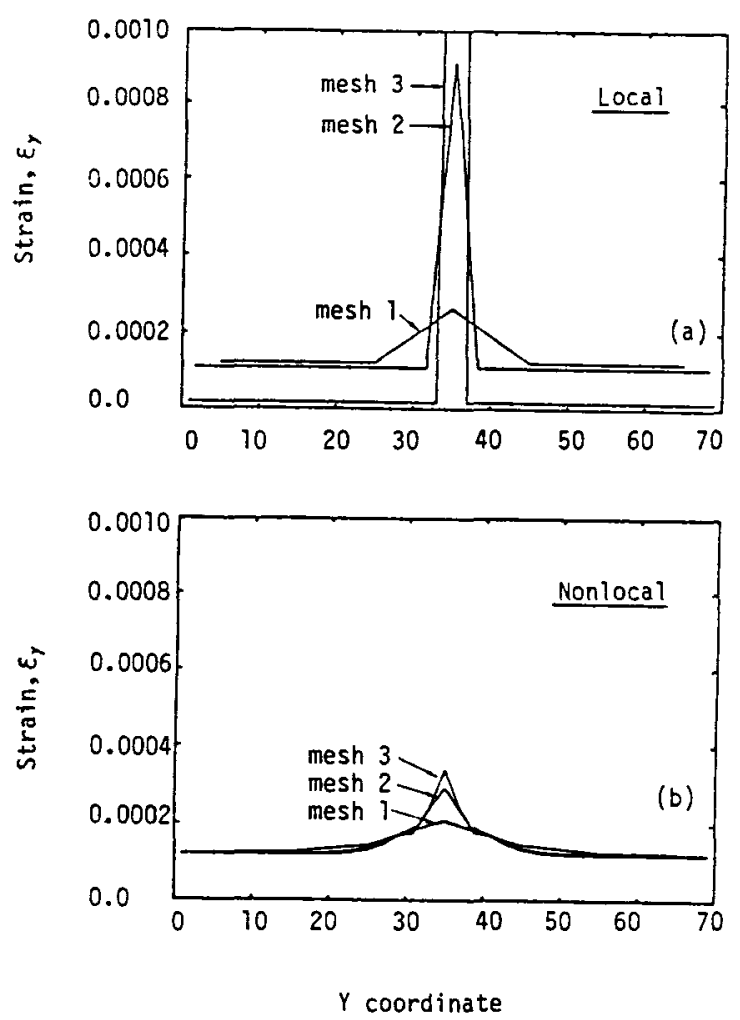

Figure 5. Profiles of vertical normal strain at vertical line of symmetry across strain-softening zone obtained for various meshes with local and non-local plasticity model

\section{EXAMPLE II: COMPRESSION SOFTENING DUE TO TUNNEL EXCAVATION IN GROUTED SOIL}

The present non-local model was applied to evaluate the safety of tunnel excavation in a softening soil, with particular reference to the construction of a subway in a non-cohesive granular soil stabilized by cement grouting. To make it possible to exacavate the tunnel by machines, the soil in the region of the tunnel is stabilized by injecting cement grout into bores drilled from the surface. During the excavation the surface of the excavation can be left unlined, which makes this construction miethod highly economical and susceptible to mechanization. Shortly after excavation, after the machine moves away, the tunnel surface is sprayed with fine concrete and later a relatively light concrete lining is cast in situ. The bores from the surface are spaced so denseiy that the grouted regions around the adjacent bores overlay, so that a grouted monolith is created by grouting. The grouted soil resembles concrete of very low strength and is highly inhomogeneous, with ungrouted pockets at a spacing of about 50 to $100 \mathrm{~cm}$, and lumps of highly cemented stiff material.

The grouted soil mass can be treated as a continuum only on a scale sufficiently larger than the prevalent spacing of the weak pockets and highly cemented lumps. The characteristic length $l$ of the homogenizing continuum should be roughly equal to this spacing, certainly in the order of magnitude, and therefore it is quite large, much larger than, e.g. for ordinary concrete. So this is an application where the modelling by non-local continuum seems particularly appropriate. 

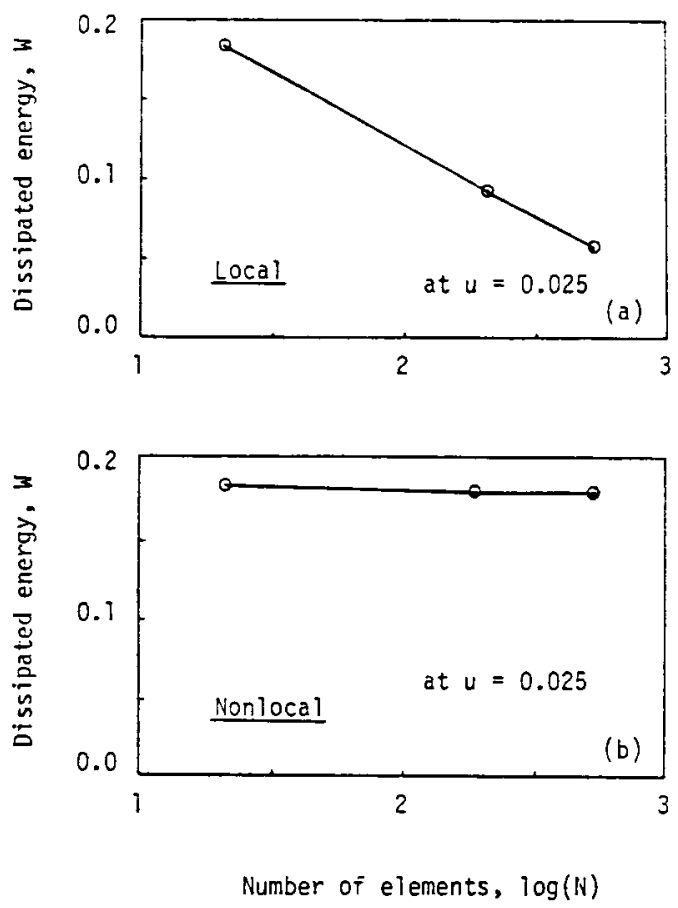

Figure 6. Convergence of energy dissipation at $u=0.025$ with increasing number of elements $N$ in the mesh. for local formulation (top) and non-local formulation (bottom)
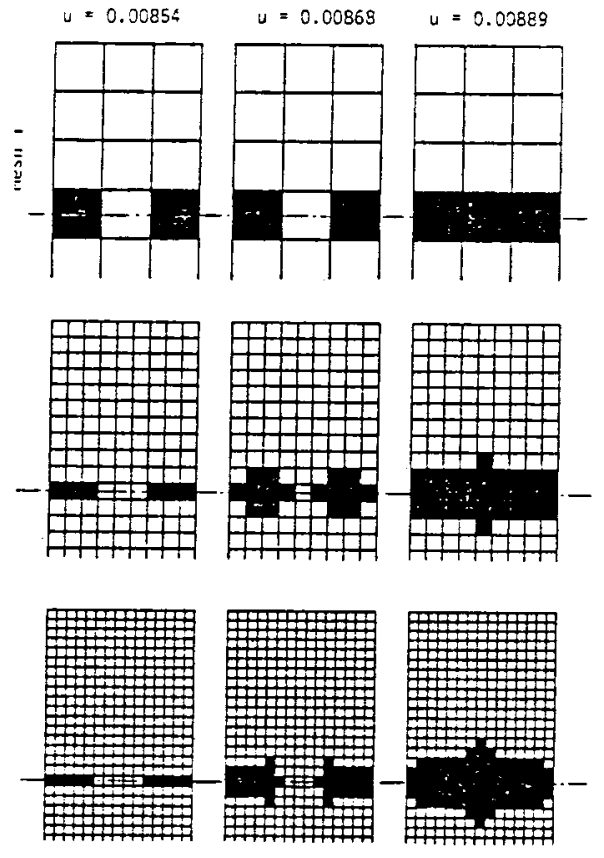

Figure 7. Softening zones at various stages of loading, for the three meshes in Figure 3 
The tunnel and the calculation results are shown in Figures 8-12. The soil is characterized by a Mohr-Coulomb yield condition (Figure 8) with a yield limit that decreases with increasing plastic strain. Prior to excavation, the vertical and horizontal normal stresses in the soil are caused solely by gravity and they increase linearly with the depth. The values of the surface tractions on the tunnel contour are calculated from the initial stress values due to gravity. Excavation is simulated by progressively decreasing the values of these surface tractions to zero. The decrease of these surface tractions causes a decrease of the lateral confining stresses as well as an increase of the vertical compression stress near the tunnel sides. This results in strain-softening behaviour in compression near the sides of the tunnel and may lead to collapse, manifested as a cave-in (or burst) of the tunnel sides.

The geometry of the tunnel is defined in Figure 9. To assess convergence of the finite element solution with compressive strain-softening, the problem was solved (on a Cray X-MP/48 supercomputer) with four two-dimensional meshes shown in Figure 10, for which the number of degrees of freedom (unknown nodal displacement components) was $N=218,608,1840$ and 3248. The characteristic length $l$ of the grouted soil, shown graphically in Figure 10, has been assumed to be $l=1.8 \mathrm{~m}$, which is roughly equal to the typical spacing of the lumps of highly grouted soil and of weakly grouted pockets as observed at the construction site. The material properties are fully defined in Figure 8, in which $E=11.4 \mathrm{ksi}, v=0.33, c=5.7 \mathrm{psi}, \phi=35^{\circ}, H=-57.8 \mathrm{psi}$.

The problem was solved both conventionally (i.e. using the usual local form of the present plasticity model with strain-softening) and by the presently proposed non-local formulation. Figure 11 shows the plots (for the four meshes used) of the horizontal inward displacement $u_{1}$ at the tunnel side surface as a function of the degree $D_{\mathrm{e}}$ of excavation (defined as the ratio of the applied surface tractions to the surface tractions that existed initially, before the excavation started). Figure 12 shows for the completed excavation $\left(D_{\mathrm{e}}=1\right)$ the profiles of the vertical normal stress $\sigma_{y}$ on a horizontal line passing roughly at the midheight of the tunnel. The minimum point on these plots corresponds to the strength limit (the peak point of the stress-strain diagram). To the right of the minimum point, the soil is in a hardening state (elastic), and to the left it is in a

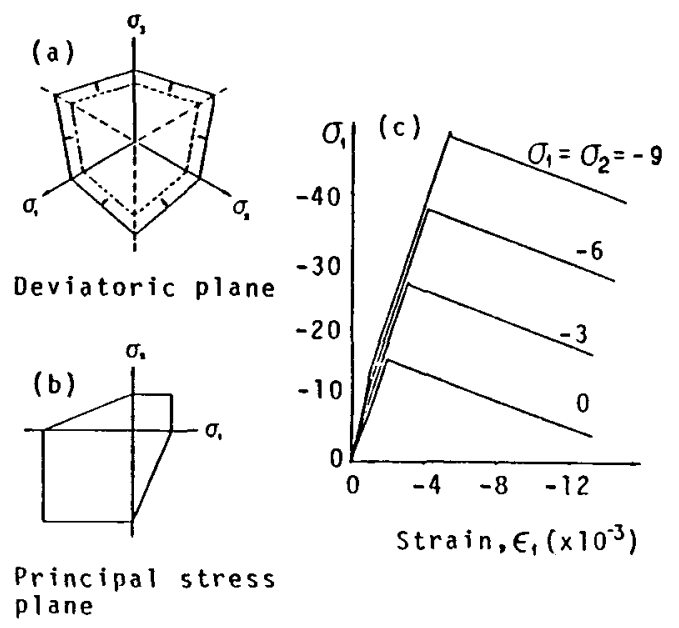

Mohr-Coulomb Yield Criteria

Figure 8. Mohr-Coulomb yield criterion with strain-softening due to yield limit degradation 

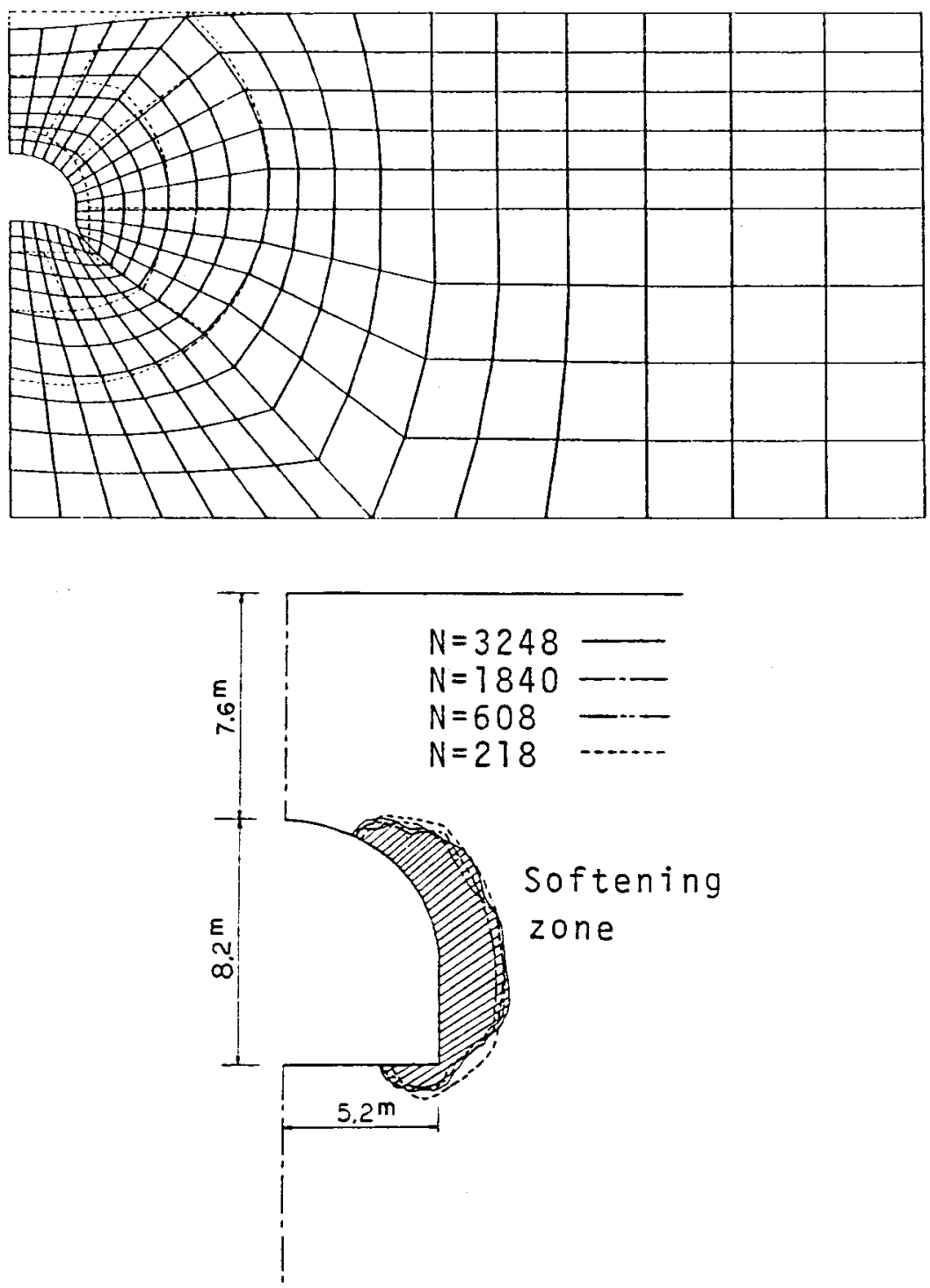

Figure 9. Boundaries of the softening zone at full tunnel excavation obtained for the four meshes shown in Figure 10, and the exaggerated deformation

state of plastic strain softening, in which the compression stress at tunnel surface is reduced to about $1 / 4$ of the strength. The lateral expansion of the compressed softening soil is quite marked, as is evident from the deformed finite element mesh shown in Figure 9(a), in which the displacements are exaggerated 70 times.

The local analysis results (Figures 11, 12 top) for the four meshes reveal a poor convergence in the softening zone at the tunnel sides, i.e. strong spurious mesh sensitivity. These results are subjective since they strongly depend on the analyst's choice of the mesh. By contrast, the nonlocal analysis results (Figures 11, 12 bottom) for the four meshes exhibit a far better convergence. 

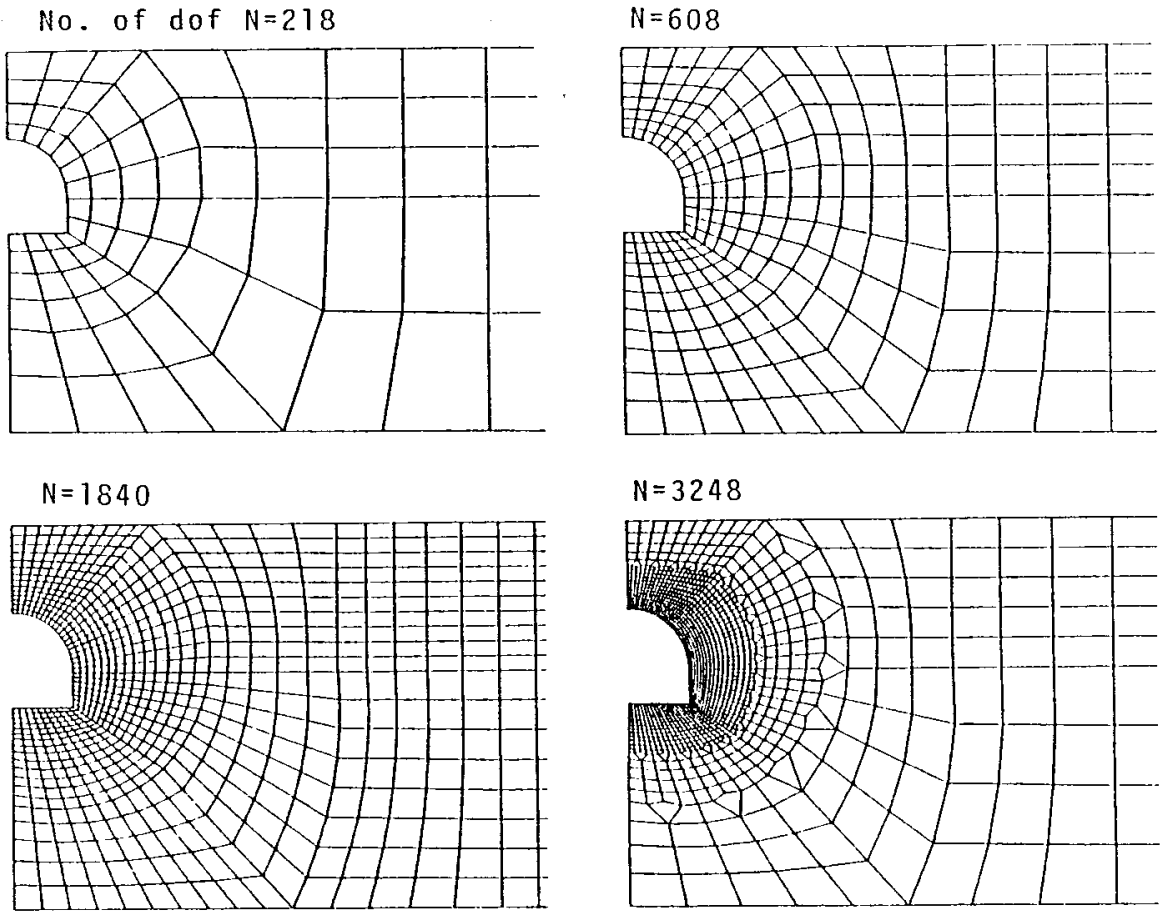

Figure 10. Finite element meshes in the analysis of a tunnel excavation in a grouted soil with a degrading yield limit

Especially the results for the meshes 3 and 4 are sufficiently close to each other for all practical purposes. So the non-local results appear to be objective.

The analysis indicates that a cave-in (burst) of the tunnel sides would not occur, i.e. the excavation without supporting the sides should be safe. In finite element calculations for various other values of material parameters, other dimensions and a different depth of the tunnel below the surface, it was often experienced that the displacements as a function of the degree of excavation would begin to increase rapidly and eventually convergence of the iterations of the loading step could not be achieved, even with the non-local formulation. Such response indicated that the tunnel would be unstable and could not be excavated safely.

Figure 9 also shows the boundaries of the softening zone obtained by non-local analysis with the four meshes. Note that all these boundaries are nearly identical, which confirms good convergence. The softening boundary is defined as the locus of the points at which the material is at the peak stress state and represents for the present constitutive model also the boundary of the elastic zone. The softening zone is in a state of a high vertical compressive strain and a high lateral tensile strain.

The numerical results confirm that the present type of non-local continuum exhibits no spurious zero-energy periodic instability modes which were encountered for other non-local models. ${ }^{6.11}$ As for the efficiency of computation, it has been experienced that for the large meshes the non-local finite element runs were usually running faster and converged more rapidly than the corresponding local ones. Apparently the fact that the non-local operator stabilizes the response improves the convergence to such an extent that it more than offsets the additional time the computer needs to evaluate the averages of plastic strains over the finite element mesh. 

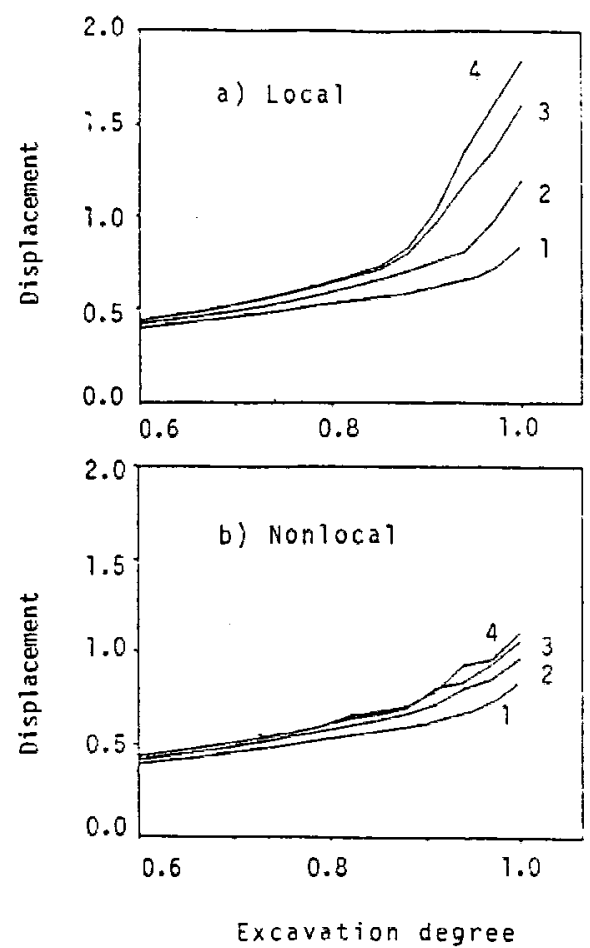

Figure 11. Response curves in the analysis of a tunnel excavation in a grouted soil with a degrading yield limit (displacement in $\mathrm{m}$ )

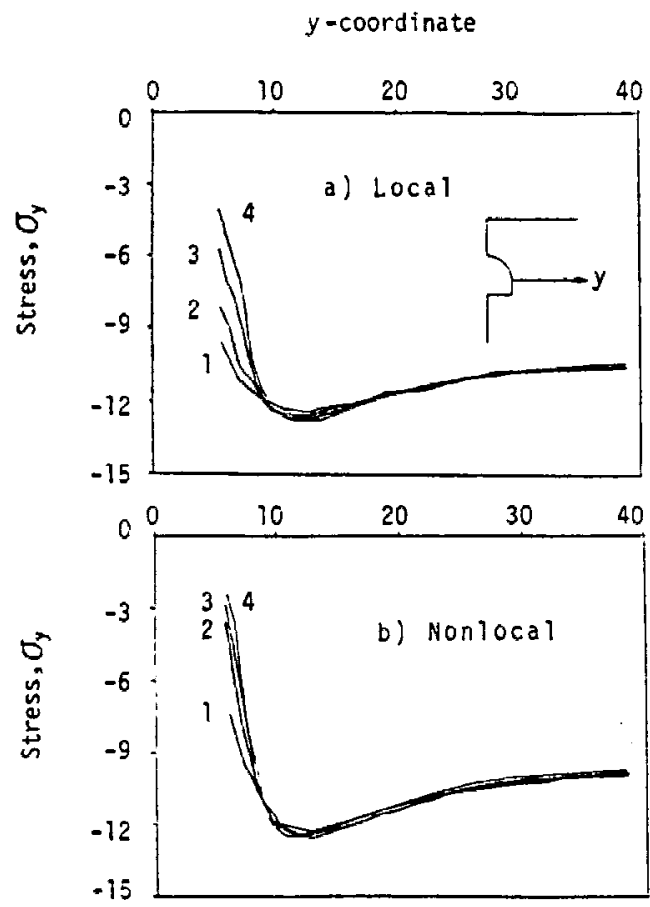

Figure 12. Stress profiles in the analysis of a tunnel excavation in a grouted soil with a degrading yield limit (stress in ton $/ \mathrm{m}^{2}=9807 \mathrm{~Pa}, y$ in $\mathrm{m}$ ) 


\section{CONCLUSIONS}

1. For strain-softening plastic materials with a degrading yield limit, localization of energy dissipation into a region of vanishing volume may be prevented by treating the plastic strains in the constitutive equation as non-local while the elastic strains remain local. This is achieved by processing the plastic strains through a spatial averaging operator, in which the characteristic length reflects the size of material inhomogeneities.

2. In contrast to the usual local approach, the finite element formulation based on the concept of non-local plastic strains exhibits correct and rapid convergence at mesh refinements and avoids spurious mesh sensitivity. The computer running times appear to be no longer than those for the local approach.

3. The new formulation has been verified for tensile as well as compressive strain softening due to yield limit degradation. It makes it possible to assess the safety against cave-in of tunnel excavation in softening soils.

\section{ACKNOWLEDGEMENT}

Partial financial support for the formulation of the basic concepts in this study was provided by U. S. Air Force Office of Scientific Research under Contract No. F49620-87-C-0030DEF with Northwestern University, monitored by Dr. Spencer T. Wu. NSF provided funds for non-local finite element programming under Grant No. MSM-8700830 to Northwestern University, and for the use of the Cray X-MP/48 supercomputer under Grant No. 10131/MSM8700830.

\section{APPENDIX-SOME DETAILS OF PLASTICITY MODEL}

The meaning of tangential plastic modulus may be clarified by considering uniaxial loading with non-zero principal stress $\sigma_{1}$ and the corresponding strain $\varepsilon_{1}$. In this case $\mathbf{D}$ reduces to Young's modulus $E$ and, from equation (11), $\Delta i=\Delta \varepsilon_{1} E /(E+H)$. The stress-strain relation becomes $\Delta \sigma_{1}=E \Delta \varepsilon_{1}[1-E /(E+H)]$, and the expression for $H$ is

$$
H=\frac{\mathrm{d} \kappa}{\mathrm{d} \varepsilon_{1}^{\mathrm{p}}}=\frac{\mathrm{d} \sigma_{1}}{\mathrm{~d} \varepsilon_{1}^{\mathrm{p}}}=\frac{\mathrm{d} \sigma_{1}}{\mathrm{~d} \varepsilon_{1}-\mathrm{d} \varepsilon_{1}^{\mathrm{e}}}=\frac{1}{E_{\mathrm{t}}^{-1}-E^{-1}}
$$

Obviously, $H>0$ signifies hardening $\left(E_{\mathrm{t}}>0\right)$ and $H<0$ softening $\left(E_{\mathrm{t}}<0\right)$.

The envelope of Mohr circles for Mohr-Coulomb material is characterized by the equation: $\tau=c-\sigma \tan \phi$, where $c=$ cohesion, $\phi=$ integral friction angle and $\sigma, \tau=$ normal and shear stress axes for Mohr's circle. This may be shown to be equivalent to the condition

$$
\sigma_{1}-\sigma_{3}=2 c \cos \phi-\left(\sigma_{1}+\sigma_{3}\right) \sin \phi
$$

where $\sigma_{1}, \sigma_{3}=$ maximum and minimum principal stresses. The principal stresses are calculated as

$$
\left\{\begin{array}{l}
\sigma_{1} \\
\sigma_{2} \\
\sigma_{3}
\end{array}\right\}=\frac{I_{1}}{3}\left\{\begin{array}{l}
1 \\
1 \\
1
\end{array}\right\}+2 \sqrt{\frac{J_{2}}{3}}\left\{\begin{array}{c}
\sin \left(\theta+60^{\circ}\right) \\
\sin \theta \\
\sin \left(\theta-60^{\circ}\right)
\end{array}\right\}
$$

in which $I_{1}=\sigma_{k k}, J_{2}=s_{i j} s_{i j} / 2, J_{3}=s_{i j} s_{j k} s_{k i} / 3, \theta=\sin ^{-1}\left(-1 \cdot 5 \sqrt{3} J_{3} J_{2}^{-3 / 2}\right) / 3, \quad-30^{\circ} \leqslant \theta \leqslant 30^{\circ} ; s_{i j}$ $=$ stress deviator. The yield surface thus becomes

$$
F(\boldsymbol{\sigma}, \kappa)=\frac{1}{3} I_{1} \sin \phi+\sqrt{J_{2}}\left(\cos \theta-\frac{1}{\sqrt{3}} \sin \theta \sin \phi\right)-\kappa
$$


where $\kappa=c \cos \phi$. To model strain-softening, the cohesion $c$ is gradually decreased.

The numerical values used in the tunnel analysis were $E=11.4 \mathrm{ksi}, v=0.33, c=5.7 \mathrm{psi}, \phi=35^{\circ}$, $H=-57.8$ psi.

The Tresca criterion is a special case of the Mohr-Coulomb criterion for $\phi=0$, and the material parameters for Example I were $E=3122 \mathrm{ksi}, v=0 \cdot 18, \sigma_{y}=2 c=0.4 \mathrm{ksi}, H=-80 \mathrm{ksi}$.

\section{REFERENCES}

1. E. C. Aifantis, 'On the microstructural origin of certain inelastic models', J. Eng. Mat. Tech., No. 106, 326-330 (1984).

2. Z. P. Bažant, 'Instability, ductility and size effect in strain-softening concrete', J. Eng. Mech. Div. ASCE, 102, 331-344; Discussions, 103, 357-358, 775-777, 104, 501-502 (1976) (based on Struct. Eng. Report No. 74-8/640, Northwestern University, Aug. 1974).

3. Z. P. Bažant, 'Crack band model for fracture of geomaterials', in Z. Eisenstein (ed.), Proc. 4th Int. Conf. on Numerical Methods in Geomechanics, Vol. 3. Edmonton, Canada, 1982, pp. 1137-1152.

4. Z. P. Bažant, 'Imbricate continuum and its variational derivation', J. Eng. Mech. Div. ASCE, 110, 1693-1712 (1984).

5. Z. P. Bažant, 'Mechanics of distributed cracking', Appl. Mech. Rev. ASME, 39, 675-705 (1985).

6. Z. P. Bažant, T. B. Belytschko and T.P. Chang, 'Continuum theory for strain-softening', J. Eng. Mech. Div. ASCE, 110, 1666-1692 (1984).

7. Z. P. Bažant and T. B. Belytschko, 'Wave propagation in a strain-softening bar: exact solution', J. Eng. Mech. Div. ASCE, 111, 381-389 (1985).

8. Z. P. Bažant and T. B. Belytschko, 'Localization and size effect', in C. S. Desai et al. (eds.), Int. Conf. on Constitutive Laws for Engineering Materials, Vol. 1, Tucson, Ar., 1987, Preprints, pp. 11-33.

9. Z. P. Bažant and L. Cedolin, 'Blunt crack band propagation in finite element analysis', J. Eng. Mech. Div. ASCE, 105, 297-315 (1979).

10. Z. P. Bažant and L. Cedolin, 'Fracture mechanics of reinforced concrete', J. Eng. Mech. Div. ASCE, 106, 1287-1306 (1980); Discussion and closure, 108, 464-471 (1982); see also Blunt crack band propagation in finite element analysis', J. Eng. Mech. Div. ASCE, 105, 297-315 (1979).

11. Z. P. Bažant and T.-P. Chang, 'Instability of nonlocal continuum and strain-averaging', J. Eng. Mech. Div. ASCE, 110, $1441-1450$ (1984).

12. Z. P. Bažant and T.-P. Chang, 'Nonlocal finite element analysis of strain-softening solids', J. Eng. Mech. Div. ASCE, $113,84-105$ (1987).

13. Z. P. Bažant, F.-B. Lin and G. Pijaudier-Cabot, 'Yield limit degradation: nonlocal continuum with local strains', in E. Onate et al. (eds.), Int. Conf. on Computational Plasticity, Barcelona, 1987, Preprints, pp. 1757-1780.

14. Z. P. Bažant and B. H. Oh (1983), 'Crack band theory for fracture of concrete', Materials and Structures, RILEM, Paris, France, Vol. 16, pp. 155-177.

15. Z. P. Bažant, J. Pan and G. Pijaudier-Cabot, 'Softening in reinforced concrete beams and frames', Report No. 86$7 / 428$ s, Center for Concrete and Geomaterials, Northwestern University, Evanston, IL, 1986.

16. Z. P. Bažant and L. Panula, 'Statistical stability effects in concrete failure', J. Eng. Mech. Div. ASCE, 104, 1195-1212 (1978).

17. Z. P. Bažant, G. Pijaudier-Cabot and J. Pan, 'Ductility, snapback size effect and redistribution in softening beams and frames', J. Struct. Eng. ASCE, 113, 2348-2364 (1987).

18. Z. P. Bažant and G. Pijaudier-Cabot, 'Modeling of distributed damage by nonlocal continuum with local strain', in A. R. Luxmore et al. (eds.), 4th Int. Conf. on Numerical Methods in Fracture Mechanics, San Antonio, Texas 1987, Preprints, pp. 411-432.

19. Z. P. Bažant and G. Pijaudier-Cabot, 'Nonlocal damage: continuum model, localization instability and convergence', Report No 87-2/428n-I, Center for Concrete and Geomaterials, Northwestern University, Evanston, IL, 1987; also J. Appl. Mech. ASME, in press.

20. Z. P. Bažant and A. Zubelewicz, 'Strain-softening in bar and beam: exact nonlocal solution', Report, Center for Concrete and Geomaterials, Northwestern University, Evanston, IL, 1986.

21. T. B. Belytschko, Z. P. Bažant, Y.W. Hyun and T.-P. Chang, 'Strain-softening materials and finite element solutions', Comp. Struct., 23, 163-180 (1986).

22. T. B. Beltyschko, X. J. Wang, Z. P. Bažant and H. Hyun, 'Transient solutions for one-dimensional problems with strain-softening', J. Appl. Mech. ASME, 54, 513-518 (1987).

23. L. Cedolin and Z. P. Bažant, 'Effect of finite element choice in blunt crack band analysis', Comp. Methods Appl. Mech. Eng., 24, 305-316 (1980).

24. A. C. Eringen and N. Ari, 'Nonlocal stress field at Griffith crack', Cryst. Latt. Amorph. Mater., 10, 33-38 (1983).

25. A. C. Eringen and D. G. B. Edelen. 'On nonlocal elasticity', Int. J. Eng. Sci., No. 10, 233-248 (1972).

26. H. Floegl and H. A. Mang, 'On tension stiffening in cracked reinforced concrete slabs and shells considering geometric and physical nonlinearity', Ing. Arch., 51, 215-242 (1981).

27. A. L. Gurson, 'Plastic flow and fracture behavior of ductile materials incorporating void nucleation, growth and interaction', Ph.D. Thesis, Brown University; see also J. Eng. Mat. Tech., 99, 2 (1975).

28. J. Hadamard, Lecons sur la Propagation des Ondes, Chapt. VI, H. Hermann et Cie Paris, France, 1903. 
29. A. Hillerborg, (1984), 'Numerical methods to simulate softening and fracture of concrete', in G. C. Sih (ed.), Fracture Mechanics Applied to Concrete Structures, Martinus Nijhoff. The Hague.

30. A. Hillerborg, M. Modéer and Petersson, P. E. 'Analysis of crack formation and crack growth in concrete by means of fracture mechanics and finite elements', Cement Concr. Res.. No. 6(6), 773-782 (1976).

31. D. D. Joseph et al., 'Hyperbolicity and change of type in the flow of viscoelastic fluids', Arch. Rational Mech. Anal. 87, 213-242 (1986).

32. I. D. Karsan and J. O. Jirsa, 'Behavior of concrete under compression loadings', J. Struct. Div. ASCE, 95, 2543-2563 (1969).

33. D. Krajcinovic, 'Constitutive equations for damaging materials, J. Appl. Mech. ASME, 50, 355-360 (1983).

34. D. Krajcinovic and G. V. Fonseka, 'The continuous damage theory of brittle materials', J. Appl. Mech. ASME, 48, 809-815 (1981).

35. E. Kröner, 'Elasticity theory of materials with long-range cohesive forces', Int. J. Solids Struct., No. 3, 731-742 (1967).

36. J. A. Krumhansl, 'Some considerations of the relations between solid state physics and generalized continuum mechanics', in E. Kröner (ed.), Mechanics of Generalized Continua, Springer-Verlag, Berlin, 1968, pp. 298-331.

37. P. Ladevèze, 'On an anisotropic damage theory' (in French), Internal Report No. 34, Laboratoire de Mécanique et Technologie, Cachan, France, 1983.

38. F. A. Leckie, 'Constitutive equations of continuum creep damage mechanics', Philos. Trans. Roy. Soc. London, Ser. A, 288, 27-47 (1978).

39. J. Lemaitre, 'How to use damage mechanics' (Division lecture presented at SMiRT7, Chicago, 1983), Internal Report No. 40, Laboratorire de Mécanique et Technologie, Ecole Normale Supérieure de l'Enseignement Technique, ParisCachan, France, 1983.

40. J. Lemaitre and J.-L. Chaboche, 'Aspect phenomenologique de la rupture par endommagement', J. Mécan. Appl. (Paris), 2, 317-365 (1978).

41. J. Lemaitre and J. L. Chaboche, Mécanique des Matériaux Solides, Dunod-Bordas, Paris, 1985.

42. R. L'Hermite and J. J. Grieu, 'Etude expérimentales récentes sur le retrait des ciments et des bétons', Annal. I.T.T.P. (Paris), 5, 494-514 (1952).

43. H. Mang and J. Eberhardsteiner, 'Collapse analysis for thin R. C. shells on the basis of a new fracture criterion', U.S.-Japan Seminar on Finite Element Analysis of Reinforced Concrete Structures, Preprints 1985, pp. 217-238.

44. J. Mazars and G. Pijaudier-Cabot, 'Continuum damage theory: application to concrete', Internal report, Laboratoire de Mecanique et Technologie, Cachan, France, Dec. 1986.

45. A. Needleman and V. Tvergaard, J. Mech. Phys. Solids, 32, 461 (1984).

46. D. R. J. Owen and E. Hinton, Finite Elements in Plasticity: Theory and Practice, Pineridge Press, Swansea, 1980.

47. S. T. Pietruszczak and Z. Mróz, 'Finite element analysis of deformation of strain-softening materials', Int. j. numer. methods eng., 17, 327-334 (1981).

48. G. Pijaudier-Cabot and Z. P. Bažant, 'Nonlocal damage theory', Report No. 86-8/428n, Center for Concrete and Geomaterials, Northwestern University, Evanston, IL.; 1986: also J. Eng. Mech. ASCE, 113, 1512-1533 (1987).

49. G. Pijaudier-Cabot and Z. P. Bażant, 'Nonlocal damage: dynamic stability and convergence', Report No $87-2 / 428 n-I I$, Center of Concrete and Geomaterials, Northwestern University, Evanston, IL, 1987; also Comp. Struct. (in press).

50. J. H. Prevost, 'Localization of deformation in elastic-plastic solids', Int. j. numer. anal. methods geomech., 8, 187-196 (1984).

51. H. E. Read and G. P. Hegemier, 'Strain-softening of rock, soil and concrete'-a review article, Mech. Mat., 3, 271-294 (1984).

52. J. R. Rice, 'The localization of plastic deformation', Proc. 14th Int. Congr. of Theoretical and Applied Mechanics Vol. 1, Delft, The Netherlands, 1976, pp. 207-220.

53. J. W. Rudnicki and J. R. Rice, 'Conditions for the localization of deformation in pressure-sensitive dilatant materials', J. Mech. Phys. Solids, 23, 371-394 (1985).

54. I. S. Sandler, 'Strain-softening for static and dynamic problems', in K. J. Willam (ed.), Proc. Symp. on Constitutive Equations: Micro, Macro and Computational Aspects, ASME Winter Annual Meeting, New Orleans, ASME, New York, 1984, pp. 217-231.

55. H. L. Schreyer and Z. Chen, (1984), 'The effect of localization on the softening behavior of structural members', in K. J. Willam (ed.), Proc. Symp. on Constitutice Equations: Micro, Macro, and Computational Aspects, ASME Winter Annual Meeting, New Orleans, ASME, New York, 1984, pp. 193-203.

56. H. L. Schreyer and Z. Chen. 'One-dimensional softening with localization', J. Appl. Mech. ASME, 53, 791-797 (1986).

57. K. J. Willam, B. Hurlbut and S. Sture, 'Experimental, constitutive and computational aspects of concrete failure' U.S.-Japan Seminar on Finite Element Analysis of Reinforced Concretes, Tokyo, 1985, Preprints, pp. 149-172.

58. Z. P. Bažant, 'Why continuum damage is nonlocal: justification by quasiperiodic microcrack array', Mech. Res. Comm., $14,407-419(1987)$ 